\title{
Birth prevalence of tetrahydrobiopterin deficiency in China: data from the national newborn screening program, 2013-2019
}

https://doi.org/10.1515/jpem-2021-0077

Received February 3, 2021; accepted March 8, 2021;

published online April 12, 2021

\begin{abstract}
Background: Tetrahydrobiopterin deficiency (BH4D), a less common form of hyperphenylalaninemia (HPA), can lead to severe developmental retardation if untreated. Little has been reported on the prevalence of BH4D among live births worldwide. This study examined its prevalence
\end{abstract}

*Corresponding authors: Kui Deng, Department of Obstetrics, National Office for Maternal and Child Health Surveillance of China, West China Second University Hospital, Sichuan University, 20, Section 3, Ren Min South Road, Chengdu, Sichuan, P.R. China; and National Center for Birth Defects Monitoring of China, West China Second University Hospital, Sichuan University, Chengdu, Sichuan, P. R. China, Phone: +8628 85501363, Fax: +86 28 85501386,

E-mail: dengkbdpc@163.com; and Xiaohong Li, National Center for Birth Defects Monitoring of China, West China Second University Hospital, Sichuan University, 17, Section 3, Ren Min South Road, Chengdu, Sichuan, P. R. China; and Department of Obstetrics, Sichuan Birth Defects Clinical Research Center, West China Second University Hospital, Sichuan University, Chengdu, Sichuan, P. R. China; and Key Laboratory of Birth Defects and Related Diseases of Women and Children (Sichuan University), Ministry of Education, Chengdu, P. R. China, Phone: +86 28 85501189, Fax: +86 28 85501386,

E-mail: lixiaohong82@scu.edu.cn

Xuelian Yuan, National Center for Birth Defects Monitoring of China, West China Second University Hospital, Sichuan University, Chengdu, Sichuan, P. R. China

Jun Zhu, National Center for Birth Defects Monitoring of China, West China Second University Hospital, Sichuan University, Chengdu,

Sichuan, P. R. China; and Department of Obstetrics, National Office for Maternal and Child Health Surveillance of China, West China Second University Hospital, Sichuan University, Chengdu, Sichuan, P. R. China

Hanmin Liu, Department of Pediatrics, West China Second University Hospital, Sichuan University, Chengdu, Sichuan, P. R. China; and Department of Obstetrics, Sichuan Birth Defects Clinical Research Center, West China Second University Hospital, Sichuan University, Chengdu, Sichuan, P. R. China

Liangcheng Xiang, Yongna Yao and Qi Li, Department of Obstetrics, National Office for Maternal and Child Health Surveillance of China, West China Second University Hospital, Sichuan University, Chengdu, Sichuan, P. R. China across China and between geographical areas within the country.

Methods: We analyzed data from the Chinese national screening program for HPA in newborns between 2013 and 2019. BH4D prevalence was examined by province, region and the entire country. Provincial-level prevalence was estimated from the number of confirmed BH4D cases and screened newborns, after adjusting for HPA-positive recall rate. Regional- and national-level prevalences were estimated by summing provincial-level prevalences after weighting them by the number of live births. A Poisson distribution was assumed in order to calculate $95 \%$ confidence intervals (CIs) for prevalence.

Results: Among 107,078,115 newborns screened for HPA in China, 380 with BH4D were identified, corresponding to a total prevalence of 3.8 per 1,000,000 live births. Prevalence was higher in eastern regions $(5.9$ per $1,000,000)$ and northern regions (4.1 per 1,000,000) of China than in southern regions $(1.6$ per $1,000,000)$ or northwestern regions $(1.7$ per $1,000,000)$. Across the entire country, 3.9\% cases of HPA were diagnosed as BH4D, and this proportion reached as high as $15.1 \%$ in the southern part of the country.

Conclusions: These first insights into BH4D prevalence across China suggest slightly higher prevalence than in other countries, and it varies substantially by region. More attention should be paid to early diagnosis and timely treatment of BH4D.

Keywords: geographical; newborn screening; prevalence; tetrahydrobiopterin deficiency.

\section{Introduction}

Hyperphenylalaninemias (HPA) are the most common amino acid metabolic diseases [1], and occasionally they occur as tetrahydrobiopterin deficiency (BH4D) [2], in which deficiency of this essential cofactor leads to decreased metabolism of phenylalanine into tyrosine [3], which can give rise to neurometabolic disorders. BH4D can arise if any of the five enzymes involved in tetrahydrobiopterin 
biosynthesis or regeneration is deficient [4]. As for the diagnosis of BH4D, it has no specific clinical manifestation as other HPA [1], and if untreated, the resulting accumulation of phenylalanine in the brain can cause severe intellectual disability as well as behavioral and psychiatric disorders [4]. Besides dietary restriction of phenylalanine, tetrahydrobiopterin, 5-hydroxytryptophan (5-HTP) and L-dopamine are often needed for the treatment of BH4D $[1,4]$. However, most of these drugs are unaffordable for patients' families in low and middle income countries [5].

HPA is an autosomal recessive disorder with varying prevalence among countries, regions and races [4]. In contrast to substantial epidemiological data on phenylketonuria [6, 7], few studies have examined the BH4D prevalence among live births due to the rarity of the disease [4]. Several studies have focused on the prevalence of BH4D among individuals with HPA, and it varied from 1 to 20\% [4, 8-12]. Only Brazil and Japan have measured BH4D prevalence among millions of live births, indicating a rate of 0.6-2.1 per 1,000,000 [13-16]. Though geographical differences in BH4D prevalence among individuals with HPA were suggested by previous local studies in China [5, 17, 18], BH4D prevalence among live births and its geographical variation remains unknown.

Here we took advantage of newborn screening for HPA, which was introduced in China in 1981 [19, 20], and for which nationwide coverage increased from $31.0 \%$ in
2006 [21] to $97.7 \%$ in 2019 , in order to estimate the total prevalence of BH4D among live births in the country. We analyzed data for 107,078,115 newborns that had been screened from 2013 to 2019. We examined the prevalence nationally and by region, which may help estimate regional burdens of disease as well as provide insights into the genetic transmission of the condition.

\section{Materials and methods}

\section{Data source}

The data used in our study came from the Chinese Newborn Screening Information System (CNBSIS), managed by the National Office of Maternal and Child Health Surveillance (NOMCHS). The data covered the period from 2013 to 2019. Until 2019, a total of 251 newborn screening centers throughout the mainland of China used the system to report basic information on the screening, diagnosis and follow-up of newborns screened for HPA (Figure 1). During this period, around $15,000,000$ newborns were screened each year in the whole country, accounting for approximately $93.4 \%$ of live births in average.

Data quality and accuracy were checked by experienced professionals in provincial newborn screening centers that are responsible for the information management of newborn screening within the jurisdiction. Incomplete forms and nonspecific records were returned to the local centers, and they need to complete or correct them. The final records and forms were then reported to the NOMCHS for data quality assessment. More details about data collection and quality control have been described elsewhere [22].

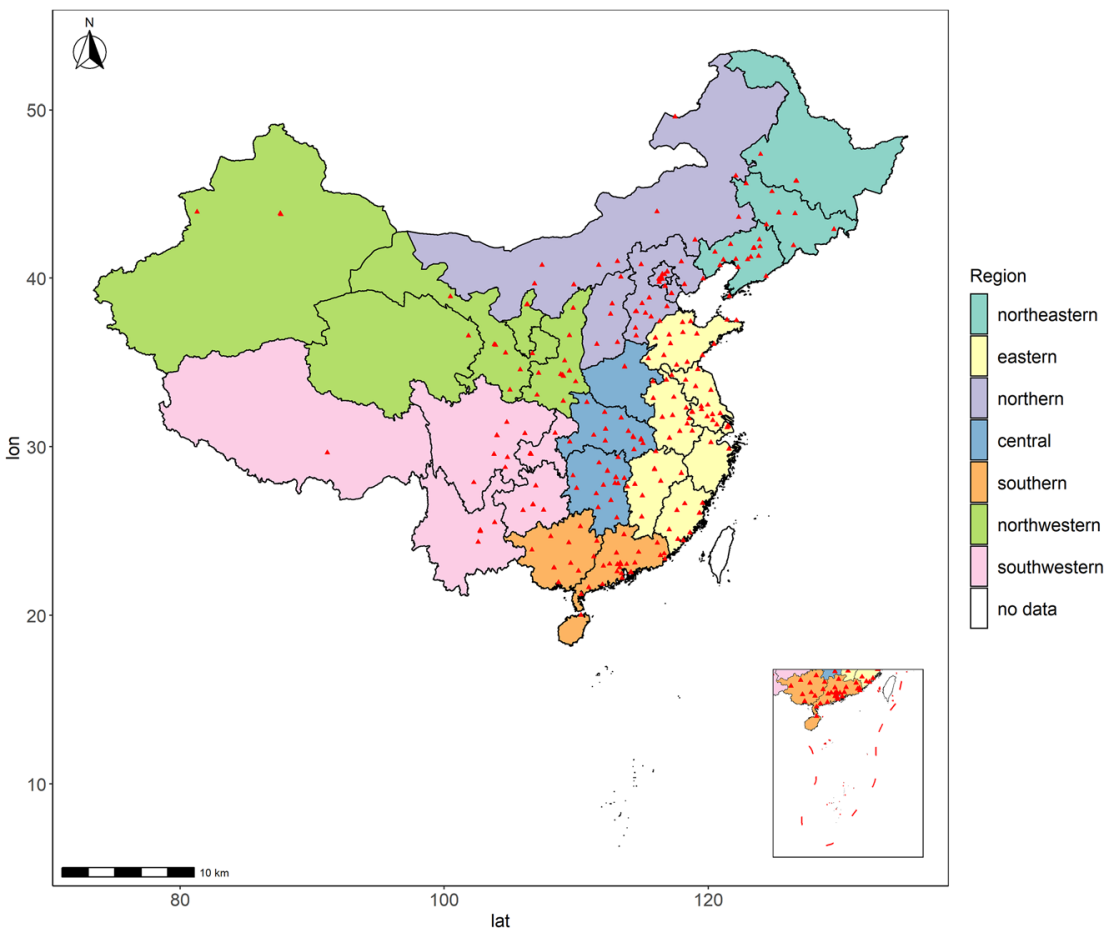

Figure 1: Distribution of newborn screening centers by province in China in 2019. 


\section{Data collection}

In each center, trained staffs were assigned to complete the online forms based on blood sample cards, laboratory test reports and medical records, including the number of newborns screened, and the demographic and clinical characteristics of infants diagnosed with HPA, which included BH4D and Phenylketonuria. These characteristics included geographical location, mother's ethnicity and age, sex, number of fetuses, gestational week, birth weight, date of birth and date of diagnosis. The present study considered only live births between 2013 and 2019 that were diagnosed with BH4D. Screened newborns throughout the entire country were included except for Tibet, where newborn screening began in 2015 and the number of live births screened from 2015 to 2019 (20,658 newborns) was too small for even one case of BH4D to be detected, which were not representative.

This study was approved by the Ethics Committee of West China Second University Hospital (No. 2015011), and performed in accordance with the 1964 Declaration of Helsinki and its subsequent amendments.

\section{BH4D diagnosis}

The procedure of the disease screening and diagnosis must be conducted in accordance with Technical Regulations For Neonatal Screening issued by Ministry of Health in 2010 [23]. HPA was diagnosed in newborns when the concentration of blood phenylalanine was higher than $120 \mu \mathrm{mol} / \mathrm{L}(2 \mathrm{mg} / \mathrm{dL})$ [23]. These newborns were then tested for urinary pterin spectrum and dihydropterin reductase activity in blood to differentially diagnose Phenylketonuria or BH4D. If necessary, the BH4 loading test was performed to assist diagnosis [23].

\section{Statistical analysis}

To explore geographic variations in $\mathrm{BH} 4 \mathrm{D}$ prevalence, the 31 provinces was divided into the following seven regions based on geography and socioeconomic status: northeastern, eastern, northern, central, southern, northwestern and southwestern [24] (Figure 1, Supplementary Table 1).

Provincial crude prevalence was reported as the number of BH4D cases identified, divided by the number of newborns screened. As the HPA-positive recall rate, defined as the proportion of newborns who had been successfully recalled for diagnosis among the newborns with HPA-positive screening results, was not $100 \%$ in most provinces, and different HPA-positive recall rates could affect the number of babies who were ultimately diagnosed, we adjusted for it to yield the adjusted provincial prevalence $p_{i}$ :

$$
\text { Adjusted } p_{i}=\frac{c}{s} \times \frac{1}{\text { HPA }- \text { positive recall rate }} \times 1000000
$$

where $c$ indicated the provincial number of BH4D cases and $s$ indicated the provincial number of newborns screened.

National and regional proportions of HPA cases that were diagnosed with BH4D were defined as the number of BH4D cases identified, divided by the number of HPA cases identified. The chi-squared test was used to assess regional differences for significance. National and regional BH4D prevalences were pooled after weighting the prevalence of each province by the number of live births:

$$
P_{a d j}=\frac{\sum_{i}^{k} w_{i} s p_{i}}{\sum_{i}^{k} w_{i} s} \times 1000000, w_{i}=\frac{b_{i}}{\sum_{i}^{k} b_{i}} \times \frac{\sum_{i}^{k} s_{i}}{s_{i}}
$$

where $k$ indicated the number of provinces in the nation or region; $w_{i}$, the weight of each province; $b_{i}$, the number of live births in each province; $s_{i}$, the number of newborns screened in each province; and $P_{a d j}$, the adjusted national prevalence or regional prevalence.

Taking the BH4D prevalence in northern China as the reference, we calculated relative risk (RR) of BH4D in each of the other regions, together with the corresponding 95\% confidence intervals (CIs) based on the natural logarithm [25]. The $95 \%$ CIs of regional or national proportions of HPA cases that were diagnosed as BH4D were estimated assuming a binominal distribution [26], while $95 \%$ CIs of provincial, regional and national prevalences were estimated assuming a Poisson distribution as the following formula [27]:

$$
\text { lower limit of } p_{a d j}=c \times\left(1-\frac{1}{9 c}-\frac{1.96}{3} \sqrt{\frac{1}{c}}\right)^{3} / s \times 1000000
$$

and

$$
\begin{aligned}
\text { upper limit of } p_{a d j}= & (c+1) \times\left(1-\frac{1}{9(c+1)}+\frac{1.96}{3} \sqrt{\frac{1}{c+1}}\right)^{3} / s \\
& \times 1000000
\end{aligned}
$$

The provincial prevalences and proportions of HPA cases that were diagnosed as BH4D were depicted on a map. All statistical analyses were performed using R version 3.6.1 (R Foundation for Statistical Computing, http://www.r-project.org).

\section{Results}

A total of 107,078,115 newborns were screened for HPA in China between 2013 and 2019, and 10,553 babies were identified with HPA, of whom 380 (3.9\%, 95\% CI 3.5-4.3) were diagnosed with BH4D. Among the BH4D cases, 50.5\% were males, and $88.7 \%$ were Han Chinese. This corresponds to a total BH4D prevalence of 3.8 (95\% CI 3.5-4.2) per 1,000,000 live births (Table 1). Only a few babies with BH4D were born before 37 weeks $(1.3 \%)$ or had low birth weight (7.6\%). Most cases of BH4D (87.9\%) occurred in women younger than 35 years (Supplementary Table 2).

As shown in Table 1, lower prevalence of BH4D among cases of HPA was observed in southern China (1.6 per $1,000,000)$ and northwestern China (1.7 per 1,000,000), while the highest prevalence was in eastern China (5.9 per 1,000,000), followed by northeastern China (5.7 per 1,000,000). After adjusting for HPA-positive recall rates and provincial-level HPA screening rates, we found that babies in eastern region were three times as likely to have BH4D as those in southern region (RR 3.7, 95\% CI 2.5-5.4).

The total proportion of BH4D cases among HPA cases was $3.9 \%$, and this proportion varied significantly across 
Table 1: BH4D prevalence among live births and among HPA cases in China, 2013-2019.

\begin{tabular}{|c|c|c|c|c|c|c|}
\hline \multirow[t]{2}{*}{ Region } & \multicolumn{3}{|c|}{ BH4D prevalence per $1,000,000$ live births } & \multicolumn{3}{|c|}{ BH4D proportion among HPA cases } \\
\hline & $\mathbf{n}$ & Rate, $95 \% \mathrm{Cl}$ & $\mathrm{RR}, 95 \% \mathrm{Cl}$ & HPA cases & $\%, 95 \% \mathrm{Cl}$ & $x^{2}, p$ \\
\hline China & 380 & $3.8(3.5,4.2)$ & - & 10,553 & $3.9(3.54 .3)$ & - \\
\hline \multicolumn{7}{|l|}{ Region } \\
\hline Northeastern & 25 & $5.7(3.7,8.3)$ & $3.5(2.1,5.9)$ & 884 & $2.8(1.7,3.9)$ & $166.9(<0.05)$ \\
\hline Eastern & 184 & $5.9(5.1,6.8)$ & $3.7(2.5,5.4)$ & 3,485 & $5.3(4.6,6.0)$ & \\
\hline Northern & 45 & $4.1(3.0,5.4)$ & $2.5(1.6,4.0)$ & 2,219 & $2.0(1.4,2.6)$ & \\
\hline Central & 57 & $3.4(2.6,4.4)$ & $2.1(1.4,3.3)$ & 1,248 & $4.6(3.4,5.8)$ & \\
\hline Southern & 25 & $1.6(1.1,2.3)$ & 1.0 & 166 & $15.1(9.7,20.5)$ & \\
\hline Northwestern & 10 & $1.7(0.9,2.8)$ & $1.1(0.5,2.0)$ & 1879 & $0.5(0.2,0.8)$ & \\
\hline Southwestern & 34 & $2.8(2.0,3.8)$ & $1.8(1.1,2.8)$ & 672 & $5.1(3.4,6.8)$ & \\
\hline
\end{tabular}

BH4D, tetrahydrobiopterin deficiency; HPA, Phenylalanine hydroxylases.

regions (Table 1). The highest proportion occurred in southern region (15.1\%), followed by eastern (5.3\%) and southwestern $(5.1 \%)$ regions. The lowest proportion was observed in northwestern region (0.5\%).

BH4D prevalence among all live births varied substantially across the provinces of China (Figure 2). Six provinces had rates $>6.0$ per $1,000,000$, among which Jiangxi in eastern China had the highest rate of 10.6 per $1,000,000$. Three provinces had prevalence $<1.0$ per $1,000,000$. Similarly, the proportion of HPA cases that were BH4D varied substantially across the provinces. Nine provinces showed BH4D proportions $>6.0 \%$. Proportions were higher in southeastern and southwestern China than in northeastern and northwestern China.

Of the 380 babies diagnosed with BH4D during the study period, most (73.4\%) were diagnosed within the second month of life, and $29.7 \%$ were diagnosed within the first month of life (Table 2). Age at diagnosis varied substantially across regions. The proportion of cases diagnosed within the first month of life were higher in southern China and lower in southwestern and northeastern China.

\section{Discussion}

This is the first study to describe the prevalence of BH4D in China using the largest national database on newborn screening for HPA in the country. We also investigated geographical variations in BH4D prevalence across regions. BH4D appears more likely to affect babies in eastern and northeastern China.

Few studies have examined BH4D prevalence. A study published in 1996 estimated the global prevalence of BH4D
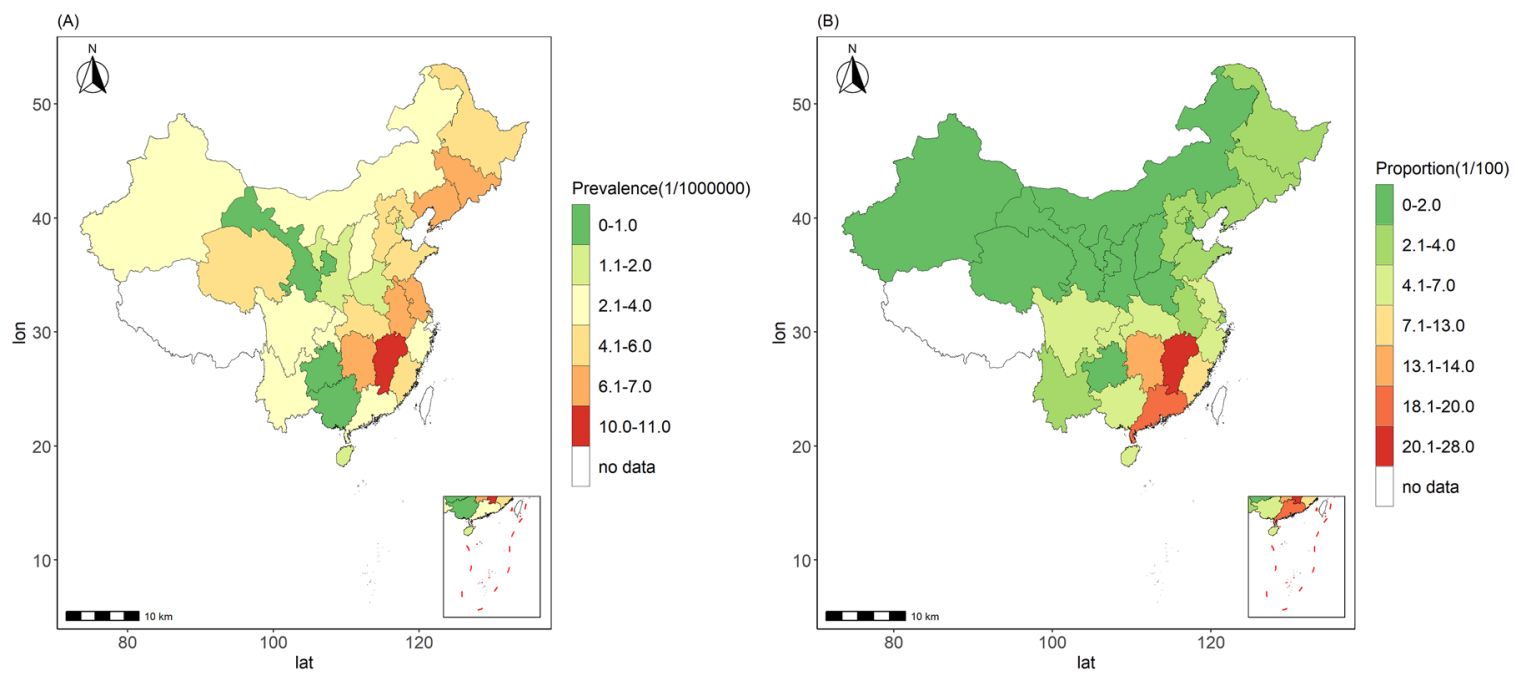

Figure 2: (A) BH4D prevalence among live births and (B) proportion of HPA cases that were diagnosed as BH4D during 2013-2019, by province in China. 
Table 2: Age at diagnosis in the 380 babies diagnosed with BH4D.

\begin{tabular}{|c|c|c|c|c|c|c|c|c|}
\hline \multirow[t]{2}{*}{ Age at diagnosis, months } & \multirow[t]{2}{*}{ Entire country } & \multicolumn{7}{|c|}{ Region of China } \\
\hline & & $\begin{array}{r}N E \\
n=25\end{array}$ & $\begin{array}{r}\text { Eastern } \\
n=184\end{array}$ & $\begin{array}{r}\begin{array}{r}\text { Northern } \\
n=45\end{array}\end{array}$ & $\begin{array}{r}\text { Central } \\
n=57\end{array}$ & $\begin{array}{r}\text { Southern } \\
n=25\end{array}$ & $\begin{array}{r}N W \\
n=10\end{array}$ & $\begin{array}{r}\text { SW } \\
n=34\end{array}$ \\
\hline$\leq 1$ & $113(29.7)$ & $3(12.0)$ & $52(28.3)$ & $18(40.0)$ & $17(29.8)$ & $17(68.0)$ & $2(20.0)$ & $4(11.8)$ \\
\hline $1-2$ & $166(43.7)$ & $14(56.0)$ & $88(47.8)$ & $19(42.2)$ & $24(42.1)$ & $5(20.0)$ & $0(0)$ & $16(47.1)$ \\
\hline$>2$ & $101(26.6)$ & $8(32.0)$ & $44(23.9)$ & $8(17.8)$ & $16(28.1)$ & $3(12.0)$ & $8(80.0)$ & $14(41.2)$ \\
\hline
\end{tabular}

Values are n, \%. BH4D, tetrahydrobiopterin deficiency; NE, northeastern; NW, northwestern; SW, southwestern.

to be 1 per 1,000,000 live births by using an international database [8]. However, it is poorly representative, as the BH4D cases in this database depended on voluntary registration by clinicians in several countries. Most of them $(68 \%)$ were Caucasian, while only $32 \%$ of them were from other countries, such as Chinese (9\%) and Japanese (6\%) [8]. A study in Minas Gerais, Brazil based on data for 19932012 estimated BH4D prevalence to be 2.1 per $1,000,000$ live births in that state [13]. Our study estimated a BH4D prevalence of 3.8 per 1,000,000 live births for the period 2013-2019, which may be higher than that reported for Minas Gerais, Brazil. Besides, we also found the BH4D prevalence in China was higher than that in Japan from 1977 to 1982 (0.6 per 1,000,000 live births). In addition to the important fact that the genetic background are different among Brazilians, Japanese and Chinese, the difference in the screening and diagnostic methods of BH4D among these countries could also help to explain such differences in BH4D prevalence. For example, the identification of BH4D in Brazil was performed only based on clinical evaluation of patients with phenylalanine-restricted diet before 2006 [13]. In Japan during 1977-1982, HPA was diagnosed in newborns when the concentration of blood phenylalanine was higher than $8 \mathrm{mg} / \mathrm{dL}$, and the differential diagnosis of BH4D was only made in patients with HPA when the effect of adequate dietary control of the blood phenylalanine level was not ideal [14]. Whereas in China, newborns with a concentration of blood phenylalanine of over $2 \mathrm{mg} / \mathrm{dL}$ would be diagnosed as HPA, and the differential diagnosis of BH4D was conducted then [23]. The reported prevalence of BH4D in Japan might be underestimated, as our data showed that approximately $29 \%$ of BH4D had a blood phenylalanine concentration of $2-8 \mathrm{mg} / \mathrm{dL}$ in the neonatal period.

Previous studies have revealed the prevalence of BH4D among individuals with HPA varies by ethnicity. For example, proportion of HPA cases diagnosed as BH4D is higher in Italy (10\%) [8], Turkey (15\%) [8] and Iran (12.3\%) [28], but it is lower in France (1.87\%) [29] and Brazil (1.71\%) [13]. Our study with a predominantly Han Chinese sample shows that even within a single country, the proportion of HPA cases diagnosed as BH4D can vary across regions. The proportion across the entire country was 3.9\% in average, which falls within the range of $1-19 \%$ reported in other researches [8], and it ranged from $0.5 \%$ in northwestern China to $15.1 \%$ in southern China. It may imply the differences between southern and northern China in the frequency of gene mutations linked to HPA [30] and in the epidemiology of the disease [31]. It may also reflect geographic differences in the availability of laboratory testing and expertise in differential diagnosis involving such a rare disease, both of which may be especially lacking in the less developed regions of western China.

Diagnosis of BH4D and initiation of treatment within the first month of life may improve prognosis [32], but fewer than $30 \%$ of BH4D cases in our system were diagnosed within that period. Given that a study in Hong Kong suggested that the screening and earlier diagnosis and treatment of 6-pyruvoyl-tetrahydropterin synthase deficiency, a subtype of BH4, among newborns can save substantial healthcare costs [33], our results suggest that it is necessary to substantially improve the timeliness of diagnosis of BH4D in China, especially in the regions where the proportion of cases diagnosed within the first month of life is relatively low.

At the same time, we must acknowledge several limitations in our study. First, we could not analyze in detail about the treatment and prognosis of these patients, since the national database did not include such data. Second, as we did not follow up all the screened newborns, the screened newborns with false-negative results could not be included in our database, which may lead to underestimation of the prevalence. Third, HPA-positive recall rate of less than $100 \%$ means that the BH4D prevalence may be underestimated. Therefore, we adjusted the prevalence by HPA-positive recall rate based on the hypothesis that the newborns with HPA-positive screening results who had not undergone diagnosis were randomly distributed in the screened newborns. Another factor that may make our BH4D prevalences underestimates is that some newborns 
who tested positive for HPA were likely referred to other specialist hospitals for diagnosis. We were unable to acquire data on patients diagnosed at specialist hospitals, so the prevalence of BH4D may have been underestimated. Future research should include analysis of BH4D diagnosis, treatment and follow-up among BH4D patients in newborn screening centers and specialist hospitals.

\section{Conclusion}

This study is the first report of nationwide prevalence of BH4D in China. The prevalence is slightly higher than that reported for other countries, and the prevalence within China varies substantially by region. Although rare, BH4D can lead to severe developmental retardation if untreated, so more attention should be paid to its early diagnosis and timely treatment.

Acknowledgments: The authors would like to thank the nurses of the obstetric and pediatric departments from the maternity hospitals for blood sample collection. The authors would also like to thank the laboratory technicians and pediatric endocrinologists from the newborn screening centers for case testing, diagnosis, treatment and follow-up. The authors are grateful to staff at the newborn screening centers for continuing collaboration and supporting with the national newborn screening database.

Research funding: This study was supported in part through the National "Thirteenth Five-Year” Key Technologies R\&D Program (Grant ID: 2017YFC1001704), the National Key Research and Development Program of China (Grant ID: 2018YFC1002200) and the Special Foundation for State Basic Research Program of China (Grant ID: 2014FY110700). Author contributions: XLY, JZ, HML, KD and XHL contributed to study design and manuscript drafting and revision. LCX, YNY and QL assisted with data review and coding. XLY, KD and XHL contributed to data analysis and interpretation. All authors reviewed and approved the final version of the manuscript. All the authors approved the submission of the final manuscript and accept responsibility for its contents.

Competing interests: The funding organization(s) played no role in the study design; in the collection, analysis, or interpretation of data; in manuscript writing; or in the decision to submit the manuscript for publication.

Ethical approval: Research involving human subjects has been approved by the Ethics Committee of West China Second University Hospital, Sichuan University.

\section{References}

1. Chinese Society of Pediatirc Endocrinology and Metabolism AgoNSoB-DPaCoCPMA. Consensus on diagnosis and treatment of hyperphenylalaninemia. Chin J Pediatr 2014;52:420-5.

2. Zurfluh MR, Giovannini M, Fiori L, Fiege B, Gokdemir Y, Baykal T, et al. Screening for tetrahydrobiopterin deficiencies using dried blood spots on filter paper. Mol Genet Metabol 2005;86(1 Suppl): S96-103.

3. Ye J, Yang Y, Yu W, Zou H, Jiang J, Yang R, et al. Demographics, diagnosis and treatment of 256 patients with tetrahydrobiopterin deficiency in mainland China: results of a retrospective, multicentre study. J Inherit Metab Dis 2013;36: 893-901.

4. Opladen T, Lopez-Laso E, Cortes-Saladelafont E, Pearson TS, Sivri HS, Yildiz Y, et al. Consensus guideline for the diagnosis and treatment of tetrahydrobiopterin (BH4) deficiencies. Orphanet J Rare Dis 2020;15:126.

5. Ye J, Liu X, Ma X, Huang X, Zhang Y, Gu X, et al. Studies on neonatal screening, clinical and gene analysis for tetrahydrobiopterin deficiency in southern Chinese. Chin J Med Genet 2001;18:92-5.

6. Shoraka HR, Haghdoost AA, Baneshi MR, Bagherinezhad Z, Zolala F. Global prevalence of classic phenylketonuria based on Neonatal Screening Program Data: systematic review and metaanalysis. Clin Exp Pediatr 2020;63:34-43.

7. Xiang L, Tao J, Deng K, Li X, Li Q, Yuan X, et al. Phenylketonuria incidence in China between 2013 and 2017 based on data from the Chinese newborn screening information system: a descriptive study. BMJ Open 2019;9:e031474.

8. Blau N, Barnes I, Dhondt JL. International database of tetrahydrobiopterin deficiencies. J Inherit Metab Dis 1996;19: 8-14.

9. Sun Y, Jiang T, Zhang J, Sun Y, Chen Y, Yang B, et al. Review of screening for neonatal hyperphenylalaninemia in Nanjing for 27 years. Chin J Prev Med 2013;16.

10. Zhao Z, Qu Y, Chen L, Yu X. Screening for phenylketonuria in 726998 neonates in Zhejiang province. J Zhejiang Univ Med Sci 2005;34.

11. Wang X, Chu Y, Maosheng GU, Wang C, Yang D, Wang X, et al. Analysis of neonatal hyperphenylalaninemia screening and therapeutic effect in Xuzhou from 2003 to 2015. Chin J Prev Med 2016;19.

12. Niu DM. Disorders of BH4 metabolism and the treatment of patients with 6-pyruvoyl-tetrahydropterin synthase deficiency in Taiwan. Brain Dev 2011;33:847-55.

13. Souza CAA, Alves MRA, Soares RDL, Kanufre VC, Rodrigues VM, Norton RC, et al. BH4 deficiency identified in a neonatal screening program for hyperphenylalaninemia. J Pediatr 2018; 94:170-6.

14. Tada K, Tateda H, Arashima S, Sakai K, Kitagawa T, Aoki K, et al. Follow-up study of a nation-wide neonatal metabolic screening program in Japan. A collaborative study group of neonatal screening for inborn errors of metabolism in Japan. Eur J Pediatr 1984;142:204-7.

15. Wang X, He Y, Jiang Y, Feng X, Zhang G, Xia Z, et al. Screening and mutation analysis of hyperphenylalaninemia in newborns from Xiamen, China. Clin Chim Acta 2019;498:161-6. 
16. Niu DM, Chien YH, Chiang CC, Ho HC, Hwu WL, Kao SM, et al. Nationwide survey of extended newborn screening by tandem mass spectrometry in Taiwan. J Inherit Metab Dis 2010;33(2 Suppl):S295-305.

17. Wang L, Yu W, Li X, He C, Chang M, Shen M, et al. Study on tetrahydrobiopterin deficiency in Northern Chinese population. Chin J Med Genet 2006;23:275-9.

18. Ye J., Qiue W-J, Han L-S, Zhou J-D Gao X-L, Gu X-F. The investigation of differential diagnostic development and incidence of tetrahydrobiopterin deficiency(Chinese). Chin J Prev Med 2009;43:128-32.

19. Gu X, Wang Z, Ye J, Han L, Qiu W. Newborn screening in China: phenylketonuria, congenital hypothyroidism and expanded screening. Ann Acad Med Singapore 2008;37(12 Suppl):107-4.

20. Zheng S, Song M, Wu L, Yang S, Shen J, Lu X, et al. China: public health genomics. Public Health Genom 2010;13:269-75.

21. Cao Y, Yuan P, Wang YP, Mao M, Zhu J. The profile of newborn screening coverage in China. J Med Screen 2009;16:163-6.

22. Deng K, He C, Zhu J, Liang J, Li X, Xie X, et al. Incidence of congenital hypothyroidism in China: data from the national newborn screening program, 2013-2015. J Pediatr Endocrinol Metab 2018;31:601-8.

23. Department of Women and Children, National Health Commission. Technical Regulations for neonatal screening; 2010. Available from: http://www.nhc.gov.cn/fys/s3585/ 201012/170f29f0c5c54d298155631b4a510df0.shtml.

24. Deng K, Zhu J, Yu E, Xiang L, Yuan X, Yao Y, et al. Incidence of inborn errors of metabolism detected by tandem mass spectrometry in China: a census of over seven million newborns between 2016 and 2017. J Med Screen 2020. https://doi.org/ 10.1177/0969141320973690.

25. Liu GW. Correct application of statistical measures in evidencebased medicine. Chin J Clin Rehabil 2003;7:359-65.
26. Zheng WH. Basic method and software operation of $95 \%$ confidence interval estimation of population rate (Chinese). Prev Med 2020;32.

27. Sever LE. Guidelines for conducting birth defects surveillance. Atlanta, GA: National Birth Defects Prevention Network; 2004.

28. Khatami S, Dehnabehoh SR, Zeinali S, Thony B, Alaei M, Salehpour S, et al. Four years of diagnostic challenges with tetrahydrobiopterin deficiencies in Iranian patients. JIMD Rep 2016. https://doi.org/10.1007/8904_2016_572.

29. Hayte JDJ. Screening of tetrahydrobiopterin deficiency among hyperphenylalaninemic patients. Excerpta Med Int Congr Ser 2002;60:165-71.

30. Yang L, Zhang Z, Ye J, Zhou Z, Shen M, Shu L, et al. Clinical study of tetrahydrobiopterin responsive phenylalanine hydroxylase deficiency in southern and northern Chinese patients(Chinese). Chin J Med Genet 2007;24:310-3.

31. Zeng W, Aiwu W, Xie X, Ouyang H, Liang J, Chen N, et al. Analysis of types and metabolic profilres of hyperphenylalaninemia. J Appl Clin Pediatr 2018;33.

32. Jaggi L, Zurfluh MR, Schuler A, Ponzone A, Porta F, Fiori L, et al. Outcome and long-term follow-up of 36 patients with tetrahydrobiopterin deficiency. Mol Genet Metabol 2008;93: 295-305.

33. Lee HH, Max CM, Poon GW-K, Wong K-Y, Lam C-W. Cost-benefit analysis of hyperphenylalaninemia due to 6-pyruvoyltetrahydropterin synthase (PTPS) deficiency: for consideration of expanded newborn screening in Hong Kong. J Med Screen 2014;21.

Supplementary Material: The online version of this article offers supplementary material (https://doi.org/10.1515/jpem-2021-0077). 